\title{
Sensitive and Prolonged Detection of Dengue Virus RNA in Whole Blood
}

\author{
Jesse J. Waggoner, ${ }^{1 \star}$ Victoria Stittleburg, ${ }^{1}$ Muktha S. Natrajan, ${ }^{1}$ Alejandra Paniagua-Avila, ${ }^{2}$ Desiree Bauer, ${ }^{3}$ Daniel Olson, ${ }^{3,4}$ \\ Hana M. El Sahly, ${ }^{5}$ Edwin J. Asturias, ${ }^{3,4}$ Evan J. Anderson, ${ }^{1,6}$ and Flor M. Munoz ${ }^{5,7}$ \\ ${ }^{1}$ Division of Infectious Diseases, Department of Medicine, Emory University, Atlanta, Georgia; ${ }^{2}$ Fundación para la Salud Integral de los \\ Guatemaltecos, FUNSALUD, Quetzaltenango, Guatemala; ${ }^{3}$ Center for Global Health, Colorado School of Public Health, Aurora, Colorado; \\ ${ }^{4}$ Division of Infectious Diseases and Epidemiology, Department of Pediatrics, University of Colorado at Denver, Aurora, Colorado; ${ }^{5}$ Departments of \\ Medicine and Molecular Virology and Microbiology, Baylor College of Medicine, Houston, Texas; ${ }^{6}$ Division of Infectious Diseases, Department of \\ Pediatrics, Emory University, Atlanta, Georgia; ${ }^{7}$ Departments of Pediatrics, Section of Infectious Diseases, and Molecular Virology and \\ Microbiology, Baylor College of Medicine, Houston, Texas
}

\begin{abstract}
Molecular detection of dengue virus (DENV) RNA from serum or plasma provides an accurate acute-phase diagnostic ( $<7$ days after symptom onset). Detection may be prolonged in whole blood, although data are limited. We tested for DENV by real-time reverse transcription-PCR in 345 paired acute-phase plasma and whole blood samples from individuals with a Flavivirus-like illness in southwestern Guatemala. In 18/18 cases with detectable DENV RNA in plasma, whole blood samples were positive and yielded similar cycle threshold values. In seven individuals with convalescent samples obtained 2-3 weeks later, DENV RNA remained detectable in whole blood but not plasma. In three additional cases, DENV RNA was only detectable in whole blood at the acute visit. In two cases, whole blood detection was linked to a virologically confirmed DENV infection 6-11 weeks earlier. Whole blood DENV RNA detection is sensitive for acute dengue infection and may remain positive for weeks to months.
\end{abstract}

Dengue virus (DENV) is the most common arboviral infection worldwide and annually results in 50-100 million symptomatic infections (dengue cases). ${ }^{1,2}$ Symptoms of dengue are nonspecific and overlap with other causes of acute febrile illness in areas of endemicity. ${ }^{2,3}$ Diagnostic confirmation relies on laboratory testing to detect 1) viral RNA or nonstructural protein 1 (NS1) antigen in acute-phase samples or 2) seroconversion of DENV IgM or IgG antibodies from the acute to convalescent phases of illness. ${ }^{2,4}$ In the acute phase, DENV RNA detection by real-time reverse transcription-PCR (rRT$\mathrm{PCR}$ ) using either serum or plasma is more accurate than either the detection of DENV NS1 antigen or DENV $\operatorname{lgM}^{2,5}$ Whole blood is listed as an optional specimen type for DENV molecular testing in the 2009 WHO guidelines, although scant data are available regarding DENV RNA detection in specimen types other than serum or plasma. ${ }^{2,6-9}$ Based on studies of Zika virus (ZIKV) and West Nile virus, two related flaviviruses, whole blood may provide sensitive and prolonged DENV RNA detection compared with serum or plasma. ${ }^{10-14}$ In the current study, we evaluated DENV detection by rRT-PCR in acuteand convalescent-phase samples collected in a region endemic for DENV in southwestern Guatemala.

Clinical samples were obtained as part of a prospective study of the neurodevelopmental outcomes of ZIKV and DENV infections among infants and young children, sponsored by the U.S. NIH. ${ }^{15,16}$ In brief, study participants included mother-infant pairs, enrolled when the babies were $<3$ months old, siblings in the household who were $<5$ years old at study entry, and a separate cohort of children who had been enrolled in a prior study of dengue seroprevalence. Participants were enrolled from June 15, 2017 to June 14, 2018 , and followed up with weekly phone calls and regular study visits for one year to surveil for acute febrile illnesses and monitor pediatric neurological development. The study

\footnotetext{
*Address correspondence to Jesse J. Waggoner, Division of Infectious Diseases, Department of Medicine, Emory University, 1760 Haygood Dr. NE, Rm. E-169, Atlanta, GA 30322. E-mail: jjwaggo@ emory.edu
}

protocol was reviewed and approved by the Institutional Review Board at Baylor College of Medicine and Emory University, the Colorado Multiple Institutional Review Board, and the Ethics Review Committee of the Ministry of Public Health and Social Welfare in Guatemala.

Ethylenediaminetetraacetic acid (EDTA) whole blood samples were collected at study enrollment from all participants, and acute illness EDTA whole blood samples were obtained within 7 days of symptom onset from participants who developed a Flavivirus-like illness (FLI). An FLI was defined as having two or more of the following signs or symptoms for $>1$ day: fever $>38.0^{\circ} \mathrm{C}$, rash, conjunctivitis, arthralgia, myalgia, and/or periarticular edema. During the acute illness visit, EDTA whole blood was collected. Two 200- $\mu \mathrm{L}$ aliquots of whole blood were prepared, and the remainder of the sample was then processed for plasma. Aliquots of whole blood and plasma were stored at $-80^{\circ} \mathrm{C}$ until laboratory testing.

Total nucleic acids were extracted from $200 \mu \mathrm{L}$ of plasma and $50 \mu \mathrm{L}$ of whole blood with an eMAG instrument (bioMerieux, Durham, NC) using the manufacturer-recommended protocols. Nucleic acids were eluted into $50 \mu \mathrm{L}$ of elution buffer for plasma and $25 \mu \mathrm{L}$ for whole blood. Nucleic acid extraction was immediately followed by testing in a multiplex rRT-PCR for ZIKV, chikungunya virus (CHIKV), and DENV (the "ZCD" assay) as previously described. ${ }^{17}$ Dengue virus detection was confirmed using a quantitative, serotype-specific rRT-PCR. ${ }^{18,19}$ For a sample to be considered positive for DENV by rRT-PCR, DENV RNA had to be detectable using both the ZCD and serotype-specific assays. Zika virus and CHIKV infections were not detected in the ZCD assay from any tested specimen.

Paired plasma and whole blood were tested from 345 FLls, which included $236 \mathrm{FLI}$ events (68.4\%) among children and 109 (31.6\%) among the mothers in the cohort. Dengue virus RNA was detected in 18 acute illness plasma samples (5.2\%) from 18 participants, including 15 children and 3 adults. In all 18 cases, DENV RNA was also detected in the paired whole blood sample. Infections with DENV serotypes $2(n=5)$ and 3 $(n=13)$ were observed, and serotypes were concordant 
between sample types. Cycle threshold (Ct) values were similar between plasma and whole blood (Figure 1).

Seven participants also had convalescent samples collected 2-3 weeks after the acute illness visit (Table 1). In all seven cases, DENV remained detectable by $\mathrm{rRT}$-PCR from whole blood but not from plasma. As expected, the $\mathrm{Ct}$ values increased significantly between the acute- and convalescentphase visits $(P<0.001$; Table 1$)$.

Three participants experienced an FLI event from which DENV RNA was only detectable by rRT-PCR in the acute illness visit whole blood sample, and the paired plasma sample was negative. Notably, for two of three participants, this FLI event occurred after a prior DENV infection, which had been confirmed by rRT-PCR during the study period (Table 1, cases 8 and 9). During the initial FLI event, DENV RNA was detected in both plasma and whole blood. Samples from the second FLI were collected 6 and 11 weeks later, and the DENV serotype was the same during both events for these two participants. The third participant had DENV-3 detected (Ct 33.31) only from whole blood at an acute illness visit. Convalescent samples were not available from this illness, and the participant did not present with another FLI during the study period.

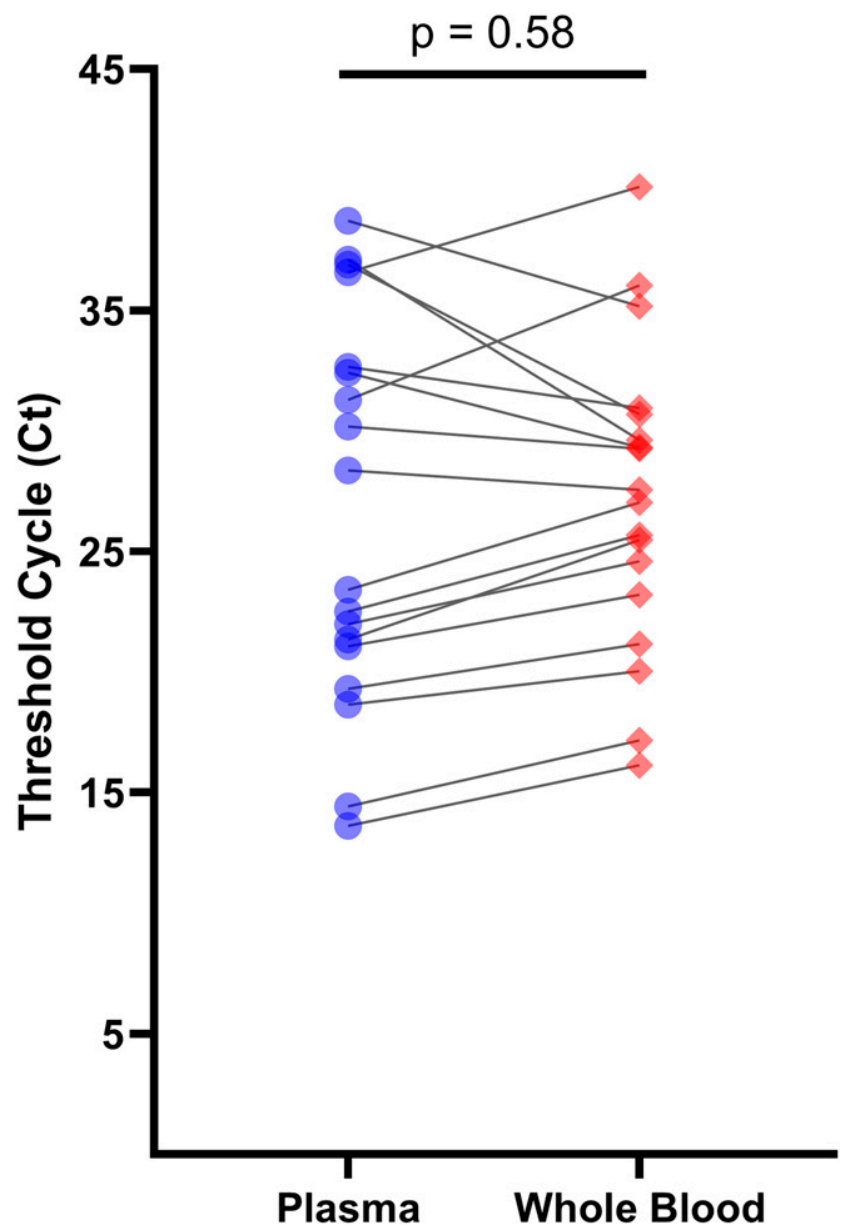

FIGURE 1. Dengue virus threshold cycle (Ct) values were similar from paired, acute-illness plasma (blue circles), and whole blood (red diamonds). Cycle threshold values were compared by paired $t$-test (GraphPad Prism software, version 8.0.1). This figure appears in color at www.ajtmh.org.
TABLE 1

Threshold cycle values from acute and convalescent illness whole blood samples in dengue virus (DENV)-positive Flavivirus-like illnesses (FLIs)

\begin{tabular}{ccccc}
\hline Case number & Acute & Convalescent & Serotype & Interval (weeks) $^{*}$ \\
\hline 1 & 27.03 & 37.54 & DENV-3 & 2 \\
2 & 20.04 & 31.70 & DENV-3 & 2 \\
3 & 21.15 & 40.57 & DENV-3 & 3 \\
4 & 29.25 & 43.28 & DENV-3 & 3 \\
5 & 30.94 & 34.75 & DENV-2 & 3 \\
6 & 17.16 & 32.76 & DENV-3 & 3 \\
7 & 25.47 & 34.67 & DENV-2 & 3 \\
8 & 27.56 & $39.19 \dagger$ & DENV-2 & 6 \\
9 & 16.14 & $40.84 \dagger$ & DENV-2 & 11 \\
\hline
\end{tabular}

† Samples were collected during the acute illness phase of a subsequent FLI event.

To better characterize Flavivirus infection histories, plasma samples collected at the enrollment visit from study participants were tested by focus reduction neutralization testing (FRNT 50 ), as described previously. ${ }^{10,20}$ Enrollment visit whole blood and plasma samples were tested by rRT-PCR from participants with high neutralization FRNT $_{50}$ titers (inverse dilution $>800$ against any DENV serotype) to detect recent Flavivirus infections. Whole blood from 7/106 (6.6\%) additional participants with high $\mathrm{FRNT}_{50}$ titers tested positive for DENV RNA, consistent with recent infection, all with DENV-3. All participants in this subgroup were asymptomatic at the time of collection. By contrast, DENV RNA was not detected from enrollment plasma by rRT-PCR from these participants.

In our study, all participants with DENV-positive plasma samples also had DENV RNA detected from whole blood by rRT-PCR, and semiquantitative viral load estimates based on Ct values did not differ significantly between these two specimen types. These data add to the limited available literature on DENV and Flavivirus RNA detection from whole blood as 1) our study population consisted predominantly of outpatient pediatric dengue cases and 2) no specimen processing was performed before extraction from a small initial specimen volume $(50 \mu \mathrm{L})$. Previous studies that compared whole blood DENV RNA detection with other specimen types enrolled hospitalized cases, which may have higher viral loads than dengue cases that present for outpatient care. ${ }^{2,5,7,8}$ In addition, studies that have demonstrated prolonged Flavivirus RNA detection from whole blood have used more complicated specimen processing protocols and larger volumes of starting material, which may not be feasible in many locations or applicable to specimens such as dried blood spots. ${ }^{8,11-14,20}$

Previous studies have not evaluated the clinical specificity of molecular detection of DENV from acute illness phase whole blood samples. In $3 / 21$ participants (14.3\%) from the current study who presented with an acute FLI, DENV RNA was detected in whole blood but not in plasma. For two of these cases, DENV detection could be directly tied to recent, prior dengue infections that occurred 6-11 weeks earlier. Given these findings, the use of whole blood for DENV RNA detection may best be applied in specific circumstances to diagnose or better understand the epidemiology of DENV infections. In populations where DENV exposure is ongoing throughout the transmission season, whole blood may confirm dengue in patients who present $>7$ days after symptom onset, when viral RNA may no longer be detectable in serum or plasma. ${ }^{2}$ However, consideration should be given to 
specimen processing, as whole blood is a complicated specimen that cannot be extracted with many standard kits for RNA viruses. ${ }^{6,21}$ Finally, whole blood may provide a useful specimen for testing individuals who had a discrete DENV exposure, such as returned travelers who developed a compatible illness while in a region with ongoing transmission. ${ }^{22}$

In conclusion, whole blood provides sensitive detection of DENV RNA by rRT-PCR during acute febrile dengue illness and may remain positive for weeks to months after acute illness.

Received November 23, 2020. Accepted for publication February 3, 2021.

Published online March 22, 2021.

Acknowledgments: We thank the study team at the Fundación para la Salud Integral de los Guatemaltecos, FUNSALUD, Quetzaltenango, Guatemala. We are grateful to all study participants and their families. We thank Walla Dempsey, Kay Tomashek, and Gail Tauscher from the Division of Microbiology and Infectious Diseases (DMID), the National Institutes of Health (NIH), for their assistance throughout the study. We thank the Emory VTEU administrative and finance core for their support, including Dean Kleinhenz, Hannah Huston, Nadine Rouphael, and Michele Paine McCullough.

Financial support: This project has been funded in whole or in part with Federal funds from the National Institute of Allergy and Infectious Diseases (NIAID). Research was supported by a NIAID DMID Vaccine and Treatment Evaluation Unit (VTEU) award to Baylor College of Medicine (Contract no. HHSN27220130015I).

Disclosure: E. J. A has received personal fees from AbbVie, Pfizer, and Sanofi Pasteur for consulting, and his institution receives funds to conduct clinical research unrelated to this manuscript from Medlmmune, Regeneron, PaxVax, Pfizer, GSK, Merck, Novavax, SanofiPasteur, Janssen, and Micron. He also serves on a safety monitoring board for Kentucky BioProcessing, Inc. F.M. Munoz has conducted clinical research unrelated to this manuscript supported by the CDC, Novavax, Regeneron, BioCryst, Alios, Janssen, Gilead, and Merck, and is a member of the Data Safety Monitoring Committee for studies conducted by Moderna, Pfizer, Meissa Vaccines, Virometix, and the National Institutes of Health.

Authors' addresses: Jesse J. Waggoner, Victoria Stittleburg, and Evan J. Anderson, Division of Infectious Diseases, Department of Medicine, Emory University, Atlanta, GA, E-mails: jesse.waggoner@ emoryhealthcare.org, victoria.d.simmons@emory.edu, and evanderson@ emory.edu. Muktha S. Natrajan, Centers for Disease Control and Prevention, RDB, Atlanta, GA, E-mail: qdz9@cdc.gov. Alejandra Paniagua-Avila, Fundación para la Salud Integral de los Guatemaltecos, FUNSALUD, Quetzaltenango, Guatemala, E-mail: alejandra. paniagua.fsigcu@gmail.com. Desiree Bauer, Center for Global Health, Colorado School of Public Health, Aurora, CO, E-mail: desiree. bauer@cuanschutz.edu. Daniel Olson and Edwin J. Asturias, Center for Global Health, Colorado School of Public Health, Aurora, CO, and Division of Infectious Diseases and Epidemiology, Department of Pediatrics, University of Colorado at Denver, Aurora, CO, E-mails: daniel.olson@childrenscolorado.org and edwin.asturias@ childrenscolorado.org. Hana M. El Sahly, Departments of Medicine and Molecular Virology and Microbiology, Baylor College of Medicine, Houston, TX, E-mail: hana.elsahly@bcm.edu. Flor M. Munoz, Departments of Pediatrics, Section of Infectious Diseases, and Molecular Virology and Microbiology, Baylor College of Medicine, Houston, TX, E-mail: florm@bcm.edu.

\section{REFERENCES}

1. Stanaway JD et al., 2016. The global burden of dengue: an analysis from the Global Burden of Disease Study 2013. Lancet Infect Dis 16: 712-723.

2. World Health Organization, 2009. Dengue: Guidelines for Diagnosis, Treatment, Prevention and Control. Geneva, Switzerland: WHO Press.
3. Waggoner JJ et al., 2016. Viremia and clinical presentation in Nicaraguan patients infected with Zika virus, chikungunya virus, and dengue virus. Clin Infect Dis 63: 1584-1590.

4. Domingo C, Niedrig M, Teichmann A, Kaiser M, Rumer L, Jarman $R G$, Donoso-Mantke O, 2010. 2nd international external quality control assessment for the molecular diagnosis of dengue infections. PLoS Negl Trop Dis 4: e833.

5. Rojas $A$ et al., 2019. Characterization of dengue cases among patients with an acute illness, Central Department, Paraguay. PeerJ 7: e7852.

6. De Paula SO, Lopes da Fonseca BA, 2002. Optimizing dengue diagnosis by RT-PCR in IgM-positive samples: comparison of whole blood, buffy-coat and serum as clinical samples. J Virol Methods 102: 113-117.

7. Klungthong C, Gibbons RV, Thaisomboonsuk B, Nisalak A, Kalayanarooj S, Thirawuth V, Nutkumhang N, Mammen MP, Jr., Jarman RG, 2007. Dengue virus detection using whole blood for reverse transcriptase PCR and virus isolation. J Clin Microbiol 45: 2480-2485.

8. Anders KL et al., 2012. An evaluation of dried blood spots and oral swabs as alternative specimens for the diagnosis of dengue and screening for past dengue virus exposure. Am J Trop Med Hyg 87: 165-170.

9. Andries AC et al., 2015. Value of routine dengue diagnostic tests in urine and saliva specimens. PLoS Negl Trop Dis 9: e0004100.

10. El Sahly HM et al., 2019. Clinical, virologic, and immunologic characteristics of Zika virus infection in a cohort of US patients: prolonged RNA detection in whole blood. Open Forum Infect Dis 6: ofy352.

11. Murray $\mathrm{KO}$ et al., 2017. Prolonged detection of Zika virus in vaginal secretions and whole blood. Emerg Infect Dis 23: 99-101.

12. Lai L, Lee TH, Tobler L, Wen L, Shi P, Alexander J, Ewing H, Busch $M, 2012$. Relative distribution of West Nile virus RNA in blood compartments: implications for blood donor nucleic acid amplification technology screening. Transfusion 52: 447-454.

13. Lanteri MC, Lee TH, Wen L, Kaidarova Z, Bravo MD, Kiely NE, Kamel HT, Tobler LH, Norris PJ, Busch MP, 2014. West Nile virus nucleic acid persistence in whole blood months after clearance in plasma: implication for transfusion and transplantation safety. Transfusion 54: 3232-3241.

14. Saa $P$ et al., 2018. Investigational testing for Zika virus among U.S. Blood donors. N Engl J Med 378: 1778-1788.

15. Colbert AM et al., 2020. Reliability and validity of an adapted and translated version of the Mullen scales of early learning (ATMSEL) in rural Guatemala. Child Care Health Dev 46: 327-335.

16. Connery AK et al., 2019. Receptive language skills among young children in rural Guatemala: the relationship between the Test de Vocabulario en Imagenes Peabody and a translated and adapted version of the Mullen Scales of Early Learning. Child Care Health Dev 45: 702-708.

17. Waggoner JJ, Gresh L, Mohamed-Hadley A, Ballesteros G, Davila MJ, Tellez Y, Sahoo MK, Balmaseda A, Harris E, Pinsky BA, 2016. Single-reaction multiplex reverse transcription PCR for detection of Zika, chikungunya, and dengue viruses. Emerg Infect Dis 22: 1295-1297.

18. Waggoner JJ et al., 2013. Single-reaction, multiplex, real-time rtPCR for the detection, quantitation, and serotyping of dengue viruses. PLoS Negl Trop Dis 7: e2116.

19. Waggoner JJ et al., 2013. Comparison of the FDA-approved CDC DENV-1-4 real-time reverse transcription-PCR with a laboratory-developed assay for dengue virus detection and serotyping. J Clin Microbiol 51: 3418-3420.

20. Lai L et al.; Emory Zika Patient Study Team, 2018. Innate, T-, and B-cell responses in acute human Zika patients. Clin Infect Dis 66: 1-10.

21. Das A, Beckham TR, Mclntosh MT, 2011. Comparison of methods for improved RNA extraction from blood for early detection of classical swine fever virus by real-time reverse transcription polymerase chain reaction. J Vet Diagn Invest 23: 727-735.

22. Jensenius $M$ et al., 2013. Acute and potentially life-threatening tropical diseases in western travelers-a GeoSentinel multicenter study, 1996-2011. Am J Trop Med Hyg 88: 397-404. 